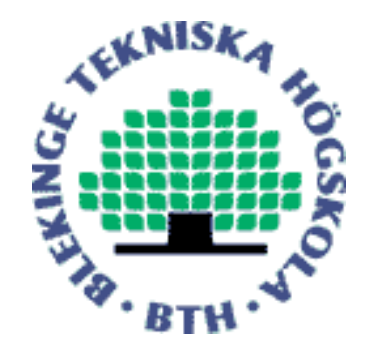

Copyright @ 2013 IEEE.

Citation for the published paper:

Title:

Suppression of Clutter in Multichannel SAR GMTI

Author:

Thomas Sjögren, Viet Vu, Mats Pettersson, Feng Wang, Daniel Murdin, Anders Gustavsson, Lars Ulander

Journal:

IEEE Transactions on Geoscience and Remote Sensing

Year:

2013

Vol:

52

Issue:

7

Pagination:

4005-4013

URL/DOI to the paper:

10.1109/TGRS.2013.2278701

This material is posted here with permission of the IEEE. Such permission of the IEEE does not in any way imply IEEE endorsement of any of BTH's products or services Internal or personal use of this material is permitted. However, permission to reprint/republish this material for advertising or promotional purposes or for creating new collective works for resale or redistribution must be obtained from the IEEE by sending a blank email message to pubs-permissions@ieee.org.

By choosing to view this document, you agree to all provisions of the copyright laws protecting it. 


\title{
Suppression of Clutter in Multichannel SAR GMTI
}

\author{
Thomas K. Sjögren, Viet T. Vu, Mats I. Pettersson, Feng Wang, \\ Daniel Murdin, Anders Gustavsson and Lars M. H. Ulander.
}

\begin{abstract}
In this paper, results of moving target detection in multichannel UHF-band Synthetic Aperture Radar (SAR) data are shown. The clutter suppression is done using Finite Impulse Response (FIR) filtering of multichannel SAR in combination with a 2-stage Fast Backprojection (FBP) algorithm to focus the moving target using relative speed. The FIR filter coefficients are chosen with the use of STAP filtering. Two parameters are used for target focusing, target speed in range and in azimuth. When the target is focused, both speed parameters of the target are found. In the experimental results, two channels were used in order to suppress clutter. In the resulting SAR images it is obvious that very strong scatterers and the forest areas have been suppressed in comparison to the moving target in the image scene. The gain obtained can be measured using SCNR gain, which is about 19dB. Another way to measure signal processing gain is the ability to suppress the strongest reflecting object in the SAR scene. The gain of target in relation to this object is $25 \mathrm{~dB}$. This shows that using UHF-band SAR GMTI for suppressing forest and increasing the target signal can work.
\end{abstract}

Index Terms-Synthetic Aperture Radar (SAR), Detection, Multichannel, MIMO radar, Space Time Adaptive Processing, STAP, clutter suppression, moving targets.

\section{INTRODUCTION}

Target detection and position estimation drove the mission requirements of the earliest radar systems.First efforts used monopulse radar was used to help to determine the position of a target. However, to be able to perform such tasks, the target should stand out in respect to the surrounding, i.e. it first has to be detected and its angle found with good precision. If a target can not be found just by scanning in angle, then knowledge of the target such as signature or Doppler generated by target motion can be used in order to detect it. To increase detection ability, many echoes can be stored and combined together for noise suppression. This suppression is due to the very short correlation time of noise and the longer correlation time for the echo of a target. However, we are not only interested in detecting an object and estimating its speed, but also in obtaining an image of the object.

Synthetic Aperture Radar (SAR) has the ability to obtain high resolution images of ground scenes including targets. Normally in a SAR image, it is hard to directly detect moving targets, especially if the clutter is strong and the target is smeared and displaced due to the movement of the target during the SAR integration time. In order to reliably detect moving targets, new signal processing algorithms are needed. Much research has been made into algorithms to detect the moving targets, to estimate their parameters and to obtain focused images of the targets.

Most SAR systems are equipped with only one antenna, and getting good results with moving target detection, parameter estimation and target focusing for single channel SAR is challenging [1]- [9]. For instance in [4], [5], the detection is made in the SAR raw data, by detecting the azimuth chirp of the moving target which is one characteristic of moving targets. In [2], [3], [7]- [9], focusing of the target in the final image is used to detect moving targets. As suggested in [6], many images can be created along the aperture and change detection applied.

With the use of two or more channels, there are more degrees of freedom to detect a moving target by suppressing radar returns from unmoving (stationary) scene elements and / or by enhancing the signatures of moving targets. For detection of moving targets in multi-channel SAR, the Displaced Phase Center Antenna (DPCA) and Along Track Interferometry (ATI) techniques have be used. DPCA basically subtracts the signal of two different antennas, as they are moved in azimuth direction [10], [11], while the ATI algorithm uses the phase difference information of the two antennas in order to detect targets [10], [12]. Many experiments with DPCA have been conducted, for instance [10], [11].

There are a number of clutter suppression approaches to enhancing moving target detection that are based on the properties of the radar sample covariance matrix [13]. Space Time Adaptive Processing (STAP) is another clutter suppression approach for moving target detection in multi-channel SAR. In STAP, instead of just subtracting two signals, statistical properties are used in order to suppress the clutter and noise. In e.g. [14], [15], STAP for SAR was suggested and in [14] results were also presented. Further results were shown in e.g. [16], [17]. Several approaches of SAR-STAP was also investigated in [18], [19]. However, in most of these experiments, the operating frequencies of the systems were at $\mathrm{X}$-band or $\mathrm{C}$-band, in which the foliage penetration ability is not as good as in UHF and VHF. The experimental results shown in this paper are within the field of STAP for lower UHF band SAR [20], thus introducing SAR-STAP results that the authors believe to be the first on low frequency SAR.

\section{FAST BACKPROJECTION}

This section gives an overview of the Fast Backprojection algorithm (FBP) [21]. FBP is a special case of the Fast Factorized Backprojection algorithm (FFBP) [22], [23]. FBP consists of one pre-stage and two processing stages. The main stages and its pseudocode are presented in Algorithm 1.

In the pre-stage, the complete aperture is split into a number of equally sized subapertures. For each subaperture, a polar grid is generated for which the step size in polar angle $\alpha$ is decided by $\Delta \cos \alpha \leq \frac{\lambda_{\min }}{2 l}$, where $l$ is sub aperture length and $\lambda_{\min }$ the shortest signal wavelength, while the step size in range $R$ is decided by $\Delta R \leq \frac{\mathrm{c}}{2 B}$ [21], where $B$ is signal 


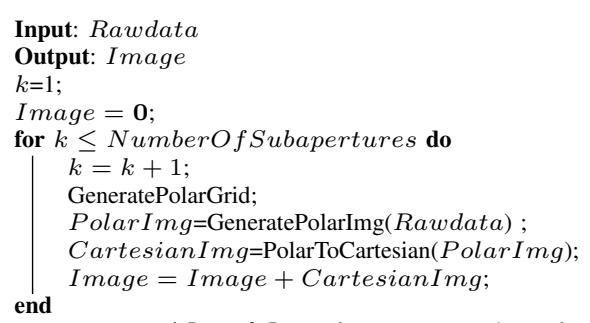

Algorithm 1: Fast Backprojection

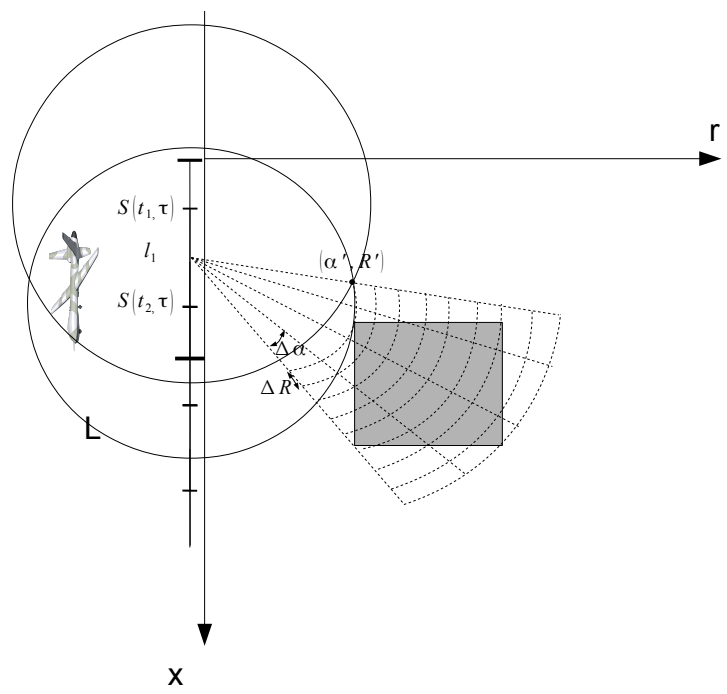

Fig. 1: The size of the sub aperture $l_{1}$ is decided based on $\Delta \alpha$. In this figure, $\alpha$ is the angle from the positive $\mathrm{x}$-axis and $\mathrm{R}$ is the distance from the centre of the sub aperture $l_{1}$ to a position in the polar image grid.

bandwidth and $\mathrm{c}$ is speed of light. Fig. 1 illustrates the polar grid which is used in FBP.

In the first stage of the algorithm, the polar image is generated by, for each position in the polar grid summing the contributions from all measurements in the sub-aperture. The generation of the polar image can be mathematically expressed by (1).

$$
\begin{aligned}
h_{k}(R, \alpha) & =\int_{t_{k}-\frac{l}{2 v_{p}}}^{t_{k}+\frac{l}{2 v_{p}}} S(t, \tau) d t \\
\tau & =\frac{2\left(\sqrt{\left(\mathrm{c}\left(t-t_{k}\right) / 2\right)^{2}+R^{2}+2 R\left(\mathrm{c}\left(t-t_{k}\right) / 2\right) \cos \alpha}\right)}{\mathrm{c}}
\end{aligned}
$$

where $t$ slow time, $t_{k}$ slow time for centre for sub aperture, $v_{p}$ is the speed of the platform, $R$ and $\alpha$ are range and angle in the polar grid, in which the angle $\alpha$ is measured from the positive azimuth axis. Here azimuth axis is defined by the sub aperture and the positive direction is defined as the flight direction. Later in the text, $h_{k}(R, \alpha)$ for a given $\alpha$ is often referred to as a beam.

In the second stage, for each subaperture, the data from the polar image is interpolated into the final Cartesian image.
Then all Cartesian images are superpositioned to generate the final SAR image.

\section{MOVING TARGET FOCUSING AND RELOCATION}

This section describes moving target focusing and how moving target focusing can be integrated with FBP. In SAR, what distinguishes a moving target from a stationary target is the different range history over the integration time. We assume a target with movement parameters given by

$$
\begin{aligned}
& x_{t c}(t)=v_{x}\left(t-t_{0}\right)+x_{0} \\
& y_{t c}(t)=v_{y}\left(t-t_{0}\right)+y_{0} \\
& z_{t c}(t)=0
\end{aligned}
$$

where $t_{0}$ is the time where the distance is at the minimum between the target and the platform and $\left(x_{0}, y_{0}\right)$ is the position of the target at minimum range.

With a platform moving at constant speed $v_{p}$ in azimuth, the range history of the target can be written as [24].

$$
R_{t}(t)=\sqrt{\gamma_{t}^{2}\left(v_{p} t-X_{t}\right)^{2}+Y_{t}^{2}}
$$

where $\gamma_{t}$ is normalized relative speed (NRS), $X_{t}$ and $Y_{t}$ azimuth and slant range image coordinates of the target.

As presented in [24], $\gamma_{t}, X_{t}$ and $Y_{t}$ are found to be

$$
\begin{gathered}
\gamma_{t}=\sqrt{\frac{\left(v_{p}-v_{x}\right)^{2}+v_{y}^{2}}{v_{p}^{2}}} \\
X_{t}=x_{0}-\frac{v_{y}}{\left(v_{p}-v_{x}\right)} y_{0} \\
Y_{t}=\sqrt{h^{2}+y_{0}^{2}\left(1+\frac{v_{y}^{2}}{\left(v_{p}-v_{x}\right)^{2}}\right)}
\end{gathered}
$$

where $\mathrm{h}$ is the flight altitude. Since we now know the targets range history, we can process the SAR image accordingly, thus focusing the target, i.e.

$$
R_{p}\left(X_{p}, Y_{p}, \gamma_{p}, t\right)=\sqrt{\gamma_{p}^{2}\left(v_{p} t-X_{p}\right)^{2}+Y_{p}^{2}}
$$

where $\gamma_{p}$ is the processing NRS, $X_{p}$ is the azimuth image coordinate and $Y_{p}$ the slant range image coordinate in order to focus all targets with $\gamma_{t}=\gamma_{p}$.

This holds for all SAR processing algorithms.

More specifically, one can modify (1) to

$$
\begin{aligned}
h_{k}(R, \alpha) & =\int_{t_{k}-\frac{l}{2 v_{p}}}^{t_{k}+\frac{l}{2 v_{p}}} S\left(t, \tau\left(\gamma_{p}\right)\right) d t \\
\tau\left(\gamma_{p}\right) & =\frac{2\left(\sqrt{\left(\mathrm{c} \gamma_{p}^{2}\left(t-t_{k}\right) / 2-R \cos \alpha\right)^{2}+R^{2} \sin ^{2} \alpha}\right)}{\mathrm{c}}
\end{aligned}
$$

The focusing can be conducted in a similar manner in the second stage of FBP, when making the transformation from polar to Cartesian image grid. 


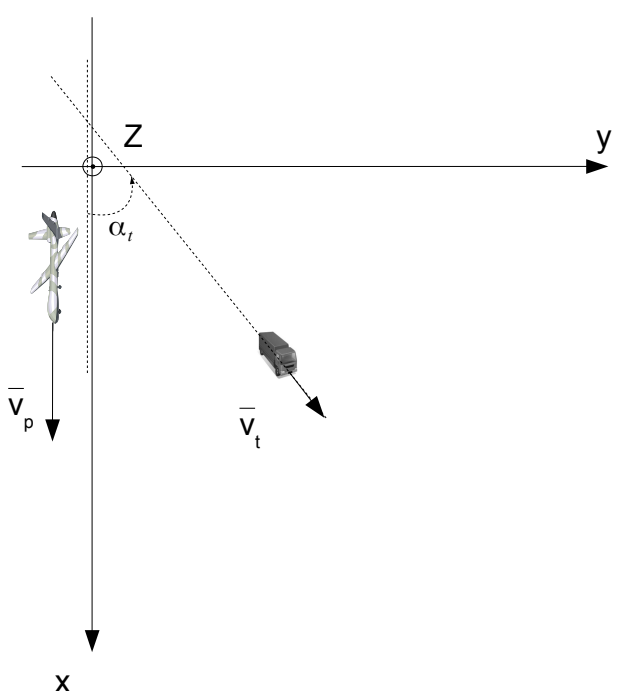

Fig. 2: The angle between the velocity vector of the platform and the target is denoted by $\alpha_{t}$.

$$
\begin{aligned}
& H\left(X_{p}, Y_{p}\right)=\sum_{k} h_{k}\left(R\left(\gamma_{p}\right), \alpha\left(\gamma_{p}\right)\right), \\
& R\left(\gamma_{p}\right)=\sqrt{\gamma_{p}^{2}\left(X_{p}-X_{c_{k}}\right)^{2}+Y_{p}^{2}}, \\
& \alpha\left(\gamma_{p}\right)=\cos ^{-1}\left(\frac{X_{p}-X_{c_{k}}}{\sqrt{\gamma_{p}^{2}\left(X_{p}-X_{c_{k}}\right)^{2}+Y_{p}^{2}}}\right)
\end{aligned}
$$

where $X_{c_{k}}$ is the azimuth position corresponding to $t=t_{k}$, i.e. the azimuth position for the origin of the k-th polar image.

If we are able to focus the target for a certain $\gamma_{p}$, the gain in SCNR is usually significant. However, we do not have information about the relation of $v_{x}$ and $v_{y}$, and since $X_{t}$ in (5) depends strongly on $v_{y}$, we need to have information about target bearing in order to find the actual $x_{0}$ and $y_{0}$ of the target.

Let us have a look at (4) again. We can see that (4) can be rewritten to the following form.

$$
\gamma_{t}=\sqrt{\left(\frac{v_{t}}{v_{p}}\right)^{2}-2 \frac{v_{t}}{v_{p}} \cos \alpha_{t}+1}
$$

where $v_{t}$ is the speed of the target, and $\alpha_{t}$ the bearing as illustrated in Fig.2.

Solving (10) for $v_{t}$, we obtain (11)

$$
v_{t}=v_{p}\left(\cos \alpha_{t}+\sqrt{\gamma_{t}^{2}+\cos ^{2} \alpha_{t}-1}\right)
$$

In section IV, equation (22) allows for estimation of the parameter $\alpha_{t}$. Having estimates of $\gamma_{t}$, and of $\alpha_{t}$, equation (11) gives us an estimate of $v_{t}$.

\section{Clutter Suppression for GMTI}

In this section, the theory for suppressing clutter in multichannel SAR is presented. First of all, in order to perform suppression, we must know what to suppress, therefore we need a model for our received signal. In this paper, the assumption is that the received signal consists of target signal, clutter and receiver noise, as formulated in (12).

$$
\mathbf{x}_{\mathbf{l}}=\mathbf{s}_{\mathbf{l}}+\mathbf{c}_{\mathbf{l}}+\mathbf{n}_{\mathbf{l}}
$$

where 1 is antenna number, $\mathbf{s}_{\mathbf{l}}$ the reflection from target, $\mathbf{c}_{\mathbf{l}}$ the received reflection from clutter and $\mathbf{n}_{\mathbf{l}}$ is the noise in the radar receiver.

In SAR, multiple radar pulses are transmitted and received with a certain pulse repetition frequency $(P R F)$ during the time when the radar system moves along a path, called the synthetic aperture. Between each pulse the radar system has moved a certain distance $\Delta l$, the noise can be assumed white and is thus decorrelated, while the clutter and the target signal still is correlated. Such a property is the basis for the development of the clutter suppression techniques such as DPCA, ATI and STAP for GMTI. This paper focuses on the STAP technique for performing clutter suppression in multichannel SAR.

In this section,we first give some background to STAP processing, and then we move to the implementation used in this paper and differences from earlier publications.

Throughout this section, $A \circ B$ means element-wise multiplications of $\mathrm{A}$ and $\mathrm{B}$, and boldface refers to vectors or matrices.

\section{A. STAP}

This subsection explains how the STAP processing was implemented in the research presented in this paper. As part of a larger processing scheme presented in this paper, STAP processing is an essential part. In Fig. 7 one finds a general overview.

In order to retrieve $\mathbf{s}_{\mathbf{l}}$ from $\mathbf{x}_{\mathbf{l}}$ in (12), an FIR filter is applied which combines range frequency samples of the channels to obtain a single filtered output signal $\mathbf{x}_{\text {filtered }}$ as presented in (13)

$$
x_{\text {filtered }}(f, k)=\sum_{l=1}^{L} x_{\text {filteredL }}(f, k, l)
$$

and

$$
\mathbf{x}_{\text {filtered } L}=\mathbf{w} \circ \mathbf{x}_{\mathbf{k}}
$$

where $\mathbf{w}$ are filter weights and $\mathbf{x}_{\mathbf{k}}$ is the measurement vector including all channels, $\mathrm{k}$ range part, or sub beam index and $\mathbf{x}_{\text {filtered } L}$ is the intermediate result where the samples for all $\mathrm{L}$ channels have been weighted. Sub beams are parts, i.e. certain fast time or range intervals of beams in the FBP polar grids, i.e. $h_{k}(R, \alpha)$ from (1) in a certain interval of $\mathrm{R}$, for a certain given $\alpha$. The reason we need to introduce $\mathrm{k}$, i.e. the sub beam index, is that that optimal filter changes along the range vector and thus must be updated. Therefore, in the implementation of STAP combined with SAR in this paper, each beam in the polar image is split in range direction into a certain number of sub-beams. 
In order to find an optimum $\mathbf{w}$, it has been suggested in [16], [25], [26] to use $\mathbf{w}$ as given in (15).

$$
\mathbf{w}_{\text {opt }}=\mathbf{u C}_{i}^{-1}
$$

In the above equation, $\mathbf{C}_{i}$ refers to the covariance matrix of the combination of all interference, in our case assumed as noise and clutter. The steering vector which is used to maximize signal strength for certain target dynamic parameters is denoted $\mathbf{u}$. Depending on the accuracy in the estimate of $\mathbf{C}_{i}$, and the noise power in relation to the covariance between the antennas, direct use of the inverse may sometimes give bad results, therefore we choose to use the Moore-Penrose pseudo inverse, thus we rewrite (15) to (16).

$$
\mathbf{w}_{\text {opt }}=\mathbf{u C}_{i}^{+}
$$

Hence, we can reformulate (13) as

$$
\mathbf{x}_{\text {filtered } L}=\frac{\mathbf{u C}_{i}^{+} \circ \mathbf{x}_{\mathbf{k}}}{N}
$$

$\mathrm{N}$ refers to a normalization factor. In this paper we chose a normalization factor according to (18). Through this choice, a constant false alarm rate (CFAR) is obtained over the image, i.e. the noise level will be equalized over the image.

$$
N=\sqrt{\mathbf{u C}_{i}^{+} \mathbf{u}^{H}}
$$

After this, each sub-beam is transformed to the Fourier domain using FFT's. Now, some steps are illustrated with data for which the complete results are shown in section $\mathrm{V}$, for which the system parameters are given in table I. In Fig. 3 the beam from a polar image is shown and in Fig. 4 one subbeam from the beam in Fig. 3. The FFT of Fig. 4 is shown in Fig. 5. As seen in Fig. 5, almost all energy of the signal is around the frequency $313 \mathrm{Mhz}$. All of these steps are always taken for both radar channels. We denote the sub-beams and their Fourier transforms by

$$
x_{k, l, f}=\mathcal{F}\left\{x_{k, l, \tau}\right\}
$$

where $\mathrm{k}$ is sub-beam number, 1 channel number, $\mathrm{f}$ frequency and $\tau$ time.

The next step is to obtain an estimate of the covariance matrix, $\mathbf{C}_{\mathbf{i}}$. This is made by calculating a weighted sum of the Fourier transforms from the sub-beams. The signal strength varies strongly between sub-beams. In order to compensate for this and obtain a good estimate for the covariance matrix, the different sub-beam FFTs are equalized.

$$
\hat{\mathbf{C}}_{\mathbf{i}}=\sum_{k} \frac{\mathbf{x}_{\mathbf{k}}{ }^{H} \mathbf{x}_{\mathbf{k}}}{\sqrt{\mathbf{x}_{\mathbf{k}} \mathbf{x}_{\mathbf{k}}{ }^{H}}}
$$

with $\mathrm{x}_{\mathbf{k}}$ given by

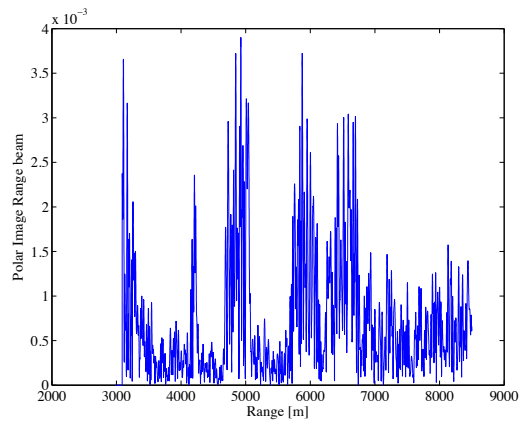

Fig. 3: This figure shows one range beam from a polar image in the middle stage of FBP.

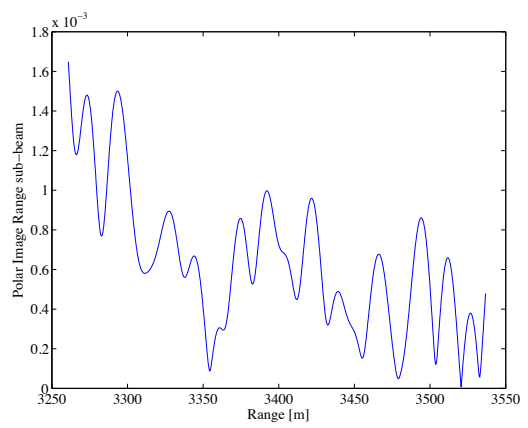

Fig. 4: After the beam in Fig. 3 is split, several sub-beams are obtained, in this figure one sub-beam of Fig. 3 is seen.

$$
\mathbf{x}_{\mathbf{k}}=\left(\begin{array}{c}
x_{k, 1, f_{\min }} \\
x_{k, 2, f_{\min }} \\
x_{k, 1, f_{\min }+d f} \\
x_{k, 2, f_{\min }+d f} \\
\vdots
\end{array}\right)^{T}
$$

After having an estimate of $\mathbf{C}_{\mathbf{i}}$, we need to define the steering vector only. In this paper, we use the steering vector for focusing a moving target with a constant speed $v_{t}$ and moving in the bearing $\alpha_{t}$. The steering vector can be found as

$$
\mathbf{u}=\left(\begin{array}{c}
1 \\
e^{-j 2 \pi f_{\text {min }} v_{t} d_{a n t} \cos \left(\alpha-\alpha_{t}\right) / v_{p}} \\
1 \\
e^{-j 2 \pi\left(f_{\text {min }}+d f\right) v_{t} d_{\text {ant }} \cos \left(\alpha-\alpha_{t}\right) / v_{p}} \\
\vdots
\end{array}\right)^{T}
$$

where $f_{\min }$ refers to lower signal frequency, $d f$ the frequency step, $d_{a n t}$ the distance between the effective phase centers of the two channels, and $v_{p}$ the platform speed.

The steering vector is in this paper used as a means to improve signal-to-interference ratio. It does so by shifting the filtered beams according to the movement of the target during the time span between the first antenna until second antenna is in same azimuth position. The steering vector is the key in being able to find target bearing as it is able to maximize 


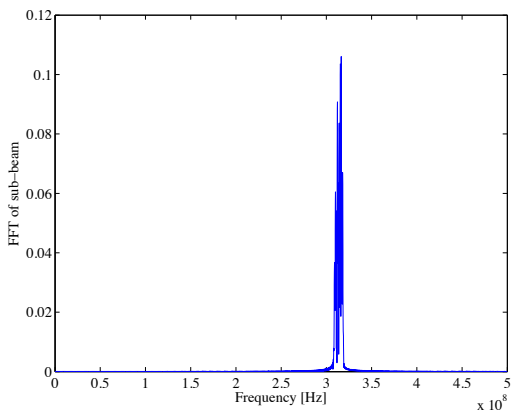

Fig. 5: After calculating the FFT of the sub-beam in Fig. 4, one obtains this sub-beam range spectrum. It is clear that almost all signal energy is concentrated around 313Mhz. Therefore, only the samples surrounding these frequencies are filtered. This helps to keep the processing burden down.

the target signal with regard to target dynamics. That means that a search in $\alpha_{t}$ for the steering vector in the filter allows for estimating $\alpha_{t}$. Furthermore, the chosen steering vector assumes target moving with constant velocity, in the same manner as for focusing using NRS.

\section{B. Integration of STAP in FBP}

Multiple methods are combined in order to perform the filtering as presented in this paper. Methods in this context refer to physical setup of radar system and also to the different signal processing techniques that are used.

First of all, with regard to the physical setup, a number of receiving antennas are used. All antennas are placed on an airborne platform with the antennas placed in along-track direction. In Fig. 6, the antenna used in the experiment presented in this paper is shown. The specific system used in the experiment in this paper is LORA [27], [28] which is a very high resolution UHF SAR system with MTI capabilities.

Secondly, with regard to SAR signal processing, a two-stage back projection algorithm is used, namely FBP which was presented in section II.

In order to suppress clutter in the SAR images, STAP is used as presented in section IV-A. This clutter suppression is conducted on the range beams in the polar images in the first stage of FBP. This is performed for each subaperture in a similar manner as in [16]. The main differences in comparison to [16] is the normalization factor used when estimating the covariance matrix, the use of FBP instead of the Local Backprojection (LBP) algorithm, and the fact that no assumptions are made with regard to dependencies within the covariance matrix. Both LBP and FBP are 2-stage implementations of FFBP, but the difference is that LBP is based on splitting the full image grid into sub-images and forming small beams over each sub-image instead of as in FBP generate beams that cover the whole image area. Fig. 7 shows the complete processing scheme for two channels from raw data to obtaining detection, focusing and speed estimation of moving targets. The pseudocode of the processing scheme is presented in Algorithm 2.

Thirdly, the hypothesis testing for target relative speed as well as the target bearing is conducted. The bearing estimation

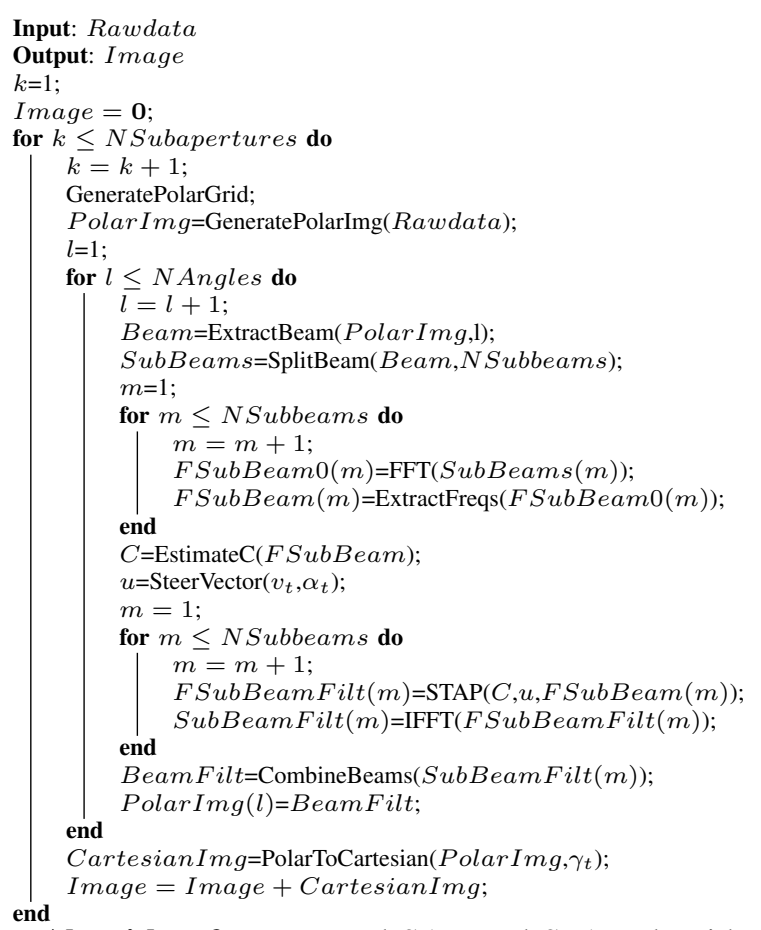

Algorithm 2: Integrated SAR and STAP algorithm

TX2 RX3 RX2 RX1 TX1

Fig. 6: In this figure, the geometrical configuration of the LORA system is shown, with two transmitting antennas and three receiving antennas. The spacing between each antenna is approximately $0.7 \mathrm{~m}$

is an integrated part of the STAP, as it is conducted by varying target parameters in the steering vector. The hypothesis testing for different relative speed is performed in the last stage of FBP, as the clutter-suppressed polar images are being backprojected onto the Cartesian image grid.

\section{RESUlts}

In this section, results using the LORA system developed by Swedish Defence Research Agency (FOI) [20] running in MTI mode with two receiving antennas and one transmitting antenna is presented. The experiment was conducted in 2005 on the island of Visingsö in the lake Vättern. The main experiment parameters can be found in Table I. In this section, coordinates in the images will be referred to as $(X, Y)$, where $X$ and $Y$ is the local azimuth and the local range respectively.

In the experiment, one truck was driven at a speed of approximately $50 \mathrm{~km} / \mathrm{h}$ on a field along a small road along the west coast of Visingsö island, while the airplanes flight path was almost orthogonal to the target path. The experiment setup is shown in Fig. 8. The target carrying a corner reflector is shown in Fig. 9. The aircraft and the truck do not move parallelly, nor perpendicularly. This leads to the target being displaced in azimuth and smeared out in the image.

In Fig. 10, the target is visible close to the position $(1500,500)$. However, the energy of the target 


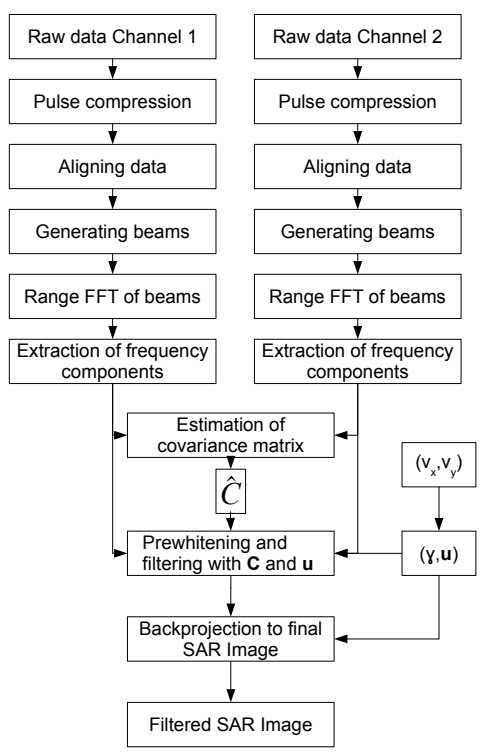

Fig. 7: In this figure, the complete processing scheme is illustrated using two radar channels, from raw data to SAR image with a focused moving target.

TABLE I: Experiment setup, Visingsö experiment site

\begin{tabular}{c|c}
\hline Parameter & Value \\
\hline \hline Centre Frequency & $313 \mathrm{MHz}$ \\
\hline Bandwidth & $12.8 \mathrm{MHz}$ \\
\hline Target Type & Truck with corner reflector \\
\hline Integration Time & $4.6 \mathrm{~s}$ \\
\hline Aircraft Speed & $130 \mathrm{~m} / \mathrm{s}$ \\
\hline Transmitter used & $\mathrm{TX} 2$ \\
\hline Receivers used & $\mathrm{RX} 1$ and RX3
\end{tabular}

is much lower compared to the strong reflecting objects such as at $(2700,500)$ which are specular reflections from large houses. In this original image, the square of peak value of the target is $2 \mathrm{~dB}$ below the average energy of the clutter. First, a certain target moving direction was guessed to be $120^{\circ}$. After this, a set of images for different NRS were created and the gain in Signal To Clutter and Noise Ratio (SCNR) was measured according to [29], as well as the gain of the target in relation to the strongest point in the image. In Fig. 11a the different measurements of SCNR gain vs NRS are shown. A peak can be seen at 1.03, corresponding to the highest SCNR gain in the process. A closer search shown in Fig. 11b was performed around 1.03, in which the maximum was found at 1.031. The gain is approximately $19 \mathrm{~dB}$.

Next step is to, based on the NRS estimate, try to focus the target better with regard to target bearing and thus obtain an estimate of $\alpha_{t}$. The results are shown in Fig. 12. Here we see that a bearing of 104 degrees appears to be a good estimate. The bearing of 104 degrees and NRS of 1.031 gives a speed of $13.87 \mathrm{~m} / \mathrm{s}$.

The highest gain in SCNR is approximately $20 \mathrm{~dB}$, with an SNR loss in the filtering at only $0.6 \mathrm{~dB}$. SNR loss is here defined as the ratio of the energy of the peak pixel of the target to the mean energy of the noise. In this best case, the gain is over $23 \mathrm{~dB}$ with regard to the strongest object in the SAR image prior to filtering. The image corresponding to these best values are found in Fig. 13, in which the target is highlighted with a red rectangle, and a zoom-in of the target signature can be seen. Please note that the target is the strongest object found in the whole image after the filtering. Also note that the clutter energy is on the same level as the noise all over the image. This indicates successful filtering. As mentioned in e.g. [27], the attenuation in the forest on UHF is less than $10 \mathrm{~dB}$, thus chances appear good to detect this target also if hidden under forest.

\section{Conclusions}

This paper presents how filtering with two-channel UHF SAR can be conducted using STAP and relative speed focusing. The paper also shows how the process can be integrated into a SAR processing algorithm named Fast Backprojection (FBP). In the proposal, the filtering step takes advantage of the fact that FBP as the first stage performs a Doppler filter. The STAP filtering is applied on the Doppler filtered beams. Results are presented from a measurement campaign with the UHF UWB SAR system LORA. The field experiment shows good results with a gain of about $20 \mathrm{~dB}$ in SCNR, while even up to $23 \mathrm{~dB}$ in gain when compared to the strongest object in the image before filtering. The strongest object here refers to object giving the strongest reflections and thus has the highest intensity in the original SAR image. It can thus be concluded that the method combined with the LORA system has good chances for success with SAR GMTI in combination with forest clutter. It should however be noted that it is not known how intermodulation effects between target and forest clutter for a target concealed in the forest would affect the SAR GMTI algorithm and therefore further experiments are needed in order to prove that a moving target hidden under forest can been detected.

\section{ACKNOWLEDGMENT}

The authors would like to thank the KK-Foundation for making this research project possible, and the support from Saab Bofors Dynamics, Saab Electronic Defence Systems and RUAG Space. Also, very special thanks to Magnus Herberthson for very valuable feedback in the process of finishing this manuscript.

\section{REFERENCES}

[1] Baumgartner, S.V., and Krieger, G. (2008) SAR traffic monitoring using time-frequency analysis for detection and parameter estimation. In Proceedings of IGARSS, Boston, MA, 7-11 July, (2008).

[2] Marques P.A.C., and Dias, J.M.B. (2005) Velocity estimation of fast moving targets using a single SAR Sensor. IEEE Transactions on Aerospace and Electronic Systems, 41, 1 (2005), 75-89.

[3] Vu, V.T., Sjögren, T.K., Pettersson, M.I., and Gustavsson, A., and Ulander, L.M.H (2010) Detection of Moving Targets by Focusing in UWB SAR - Theory and Experimental Results. IEEE Transactions on Geoscience and Remote Sensing, 48, 10 (2010), 3799-3815.

[4] Barbarossa, S. and Farina, A. (1992) Detection and imaging of moving targets with synthetic aperture radar. 2. Joint time-frequency analysis by Wigner-Ville distribution. IEE Proceedings in Radar and Signal Processing, 139, 1 (1992), 89-97. 


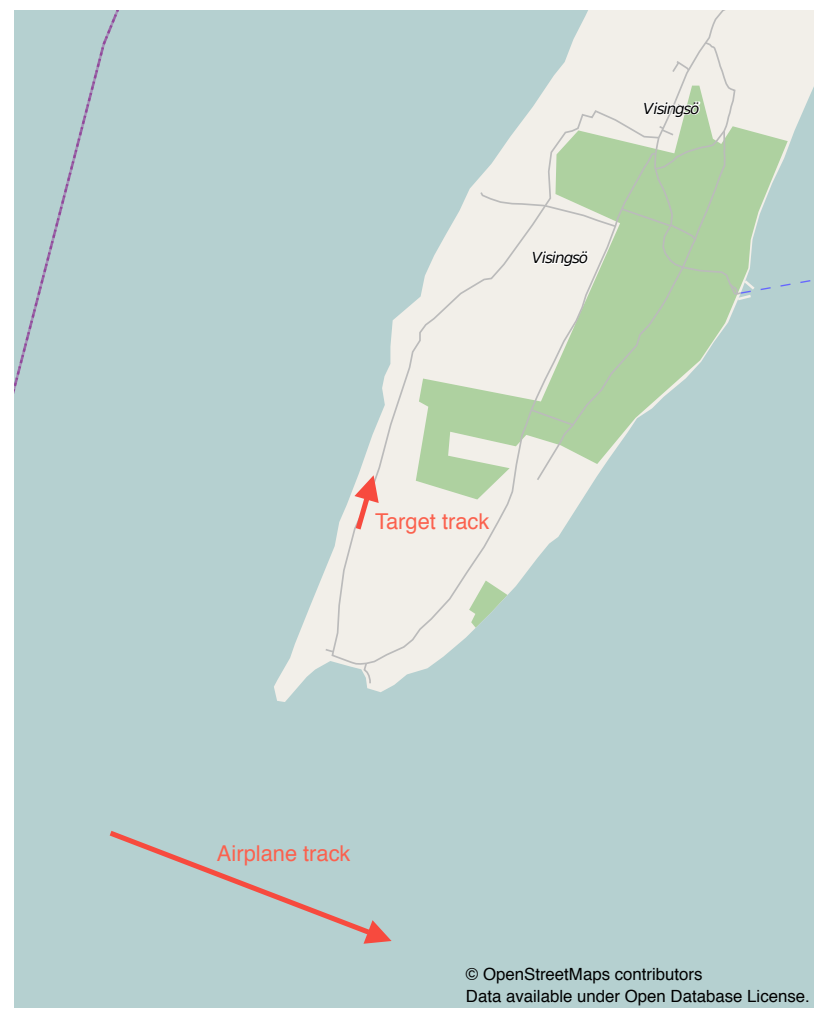

Fig. 8: The experiment area with the flight track indicated on the bottom and target path indicated along a road on the west side of Visingsö island.

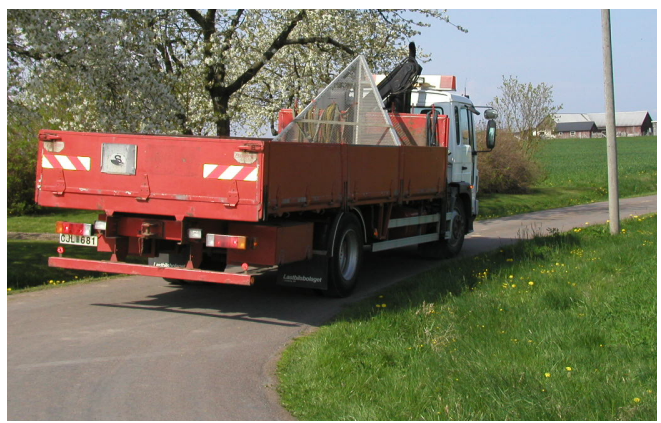

Fig. 9: The target truck with corner reflector on the roof.

[5] Zhou, F., Wu, R., Xing, M., and Bao, Z. (2007) Approach for single channel SAR ground moving target imaging and parameter estimation. IET Radar, Sonar and Navigation, 1, 1 (2007), 59-66.

[6] Zhou, H., Huang, X., Chang, Y., Zhou, Z. (2007) Single-channel UWB SAR ground moving targets detection method using change detection based on single-pass sub-aperture images. In Proceedings of Asian Pacific Conference on SAR, Huangshan, China, 5-9 November, (2007).

[7] Sjögren, T.K., Vu, V.T., Pettersson, M.I., Zepernick, H-J., Gustavsson, A. (2007) Speed estimation experiments for ground moving targets in low frequency UWB SAR. In Proceedings of IET International Radar Conference, Edinburgh, UK, 15-18 October, (2007).

[8] Jao, J.K. (2001) Theory of synthetic aperture radar imaging of a moving target. IEEE Transactions on Geoscience and Remote Sensing, 39, 9 (2001), 1984-1992.

[9] Vu, V.T., Sjögren, T.K., and Pettersson, M.I. (2008) Moving Target Detection by Focusing for Frequency Domain Algorithms in UWB

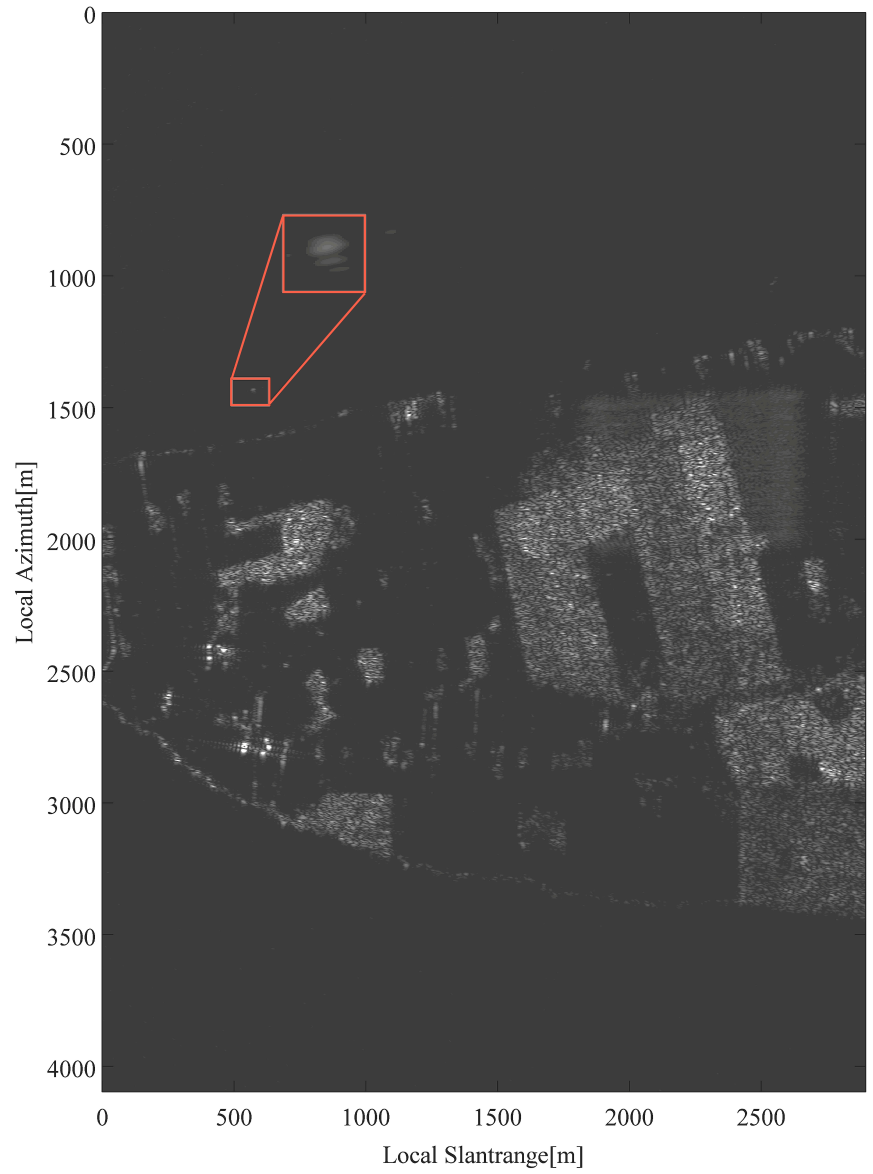

Fig. 10: In this figure, the original SAR image over the test scene is shown. The image covers $4096 \mathrm{~m}$ in azimuth and $2896 \mathrm{~m}$ in slant range.

Low Frequency SAR. In Proceedings of IGARSS, Boston, MA, 7-11 July, (2008)

[10] Gierull, C.H., Ground moving target parameter estimation for two-channel SAR. IEE Proceedings on Radar Sonar and Navigation, 153, 3 (2006), 224-233.

[11] Axelsson, S. (2004) Position correction of moving targets in SAR-Imagery. In Proceedings of SPIE - The International Society for Optical Engineering, Barcelona, Spain, 5236, 80 (2004).

[12] Suchandt, S., Runge, H., Breit, H., Steinbrecher, U., Kotenkov, A., and Balss, U. (2010) Automatic Extraction of Traffic Flows Using TerraSAR-X Along-Track Interferometry. IEEE Transactions on Geoscience and Remote Sensing, 48, 2 (2010), 807-819.

[13] Sikaneta I., Gierull C. and Chouinard J-Y. (2003) Metrics for SAR-GMTI based on Eigen decomposition of the sample covariance matrix. Proceedings of International Radar Conference, Adelaide, Australia, 3-5 Sept (2003).

[14] Ender, J.H.G. (1999) Space-Time Processing for Multichannel Synthetic Aperture Radar. Electronics and Communication Engineering Journal, 11, 1 (1999), 29-38.

[15] Ward, J. (1998) Space-time adaptive processing for airborne radar. In IEE Colloquium on Space-Time Adaptive Processing, London, UK, 6 April, (1998).

[16] Pettersson, M.I. (2004) Detection of moving targets in wideband SAR. IEEE Transactions on Aerospace and Electronic Systems, 40, 3 (2004), 780-796.

[17] Neumann, C., Meyer-Hillberg, J., Senkowski, H. (2008) STAP/GMTI analysis using SAR data from simulated scenarios. In Proceedings of International Radar Symposium 2008, Wroclaw, Poland, 21-23 May, (2008).

[18] Cerutti-Maori, D., Sikaneta I. (2010) Optimum GMTI processing for space-based SAR-GMTI systems: Theoretical derivations. Proceedings of EUSAR, Aachen, Germany, 7-10 June (2010). 


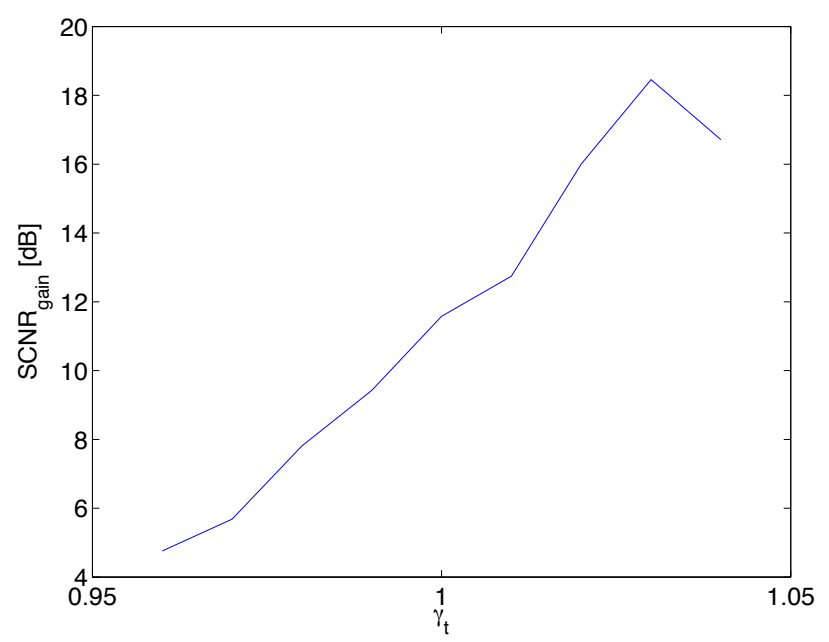

(a)

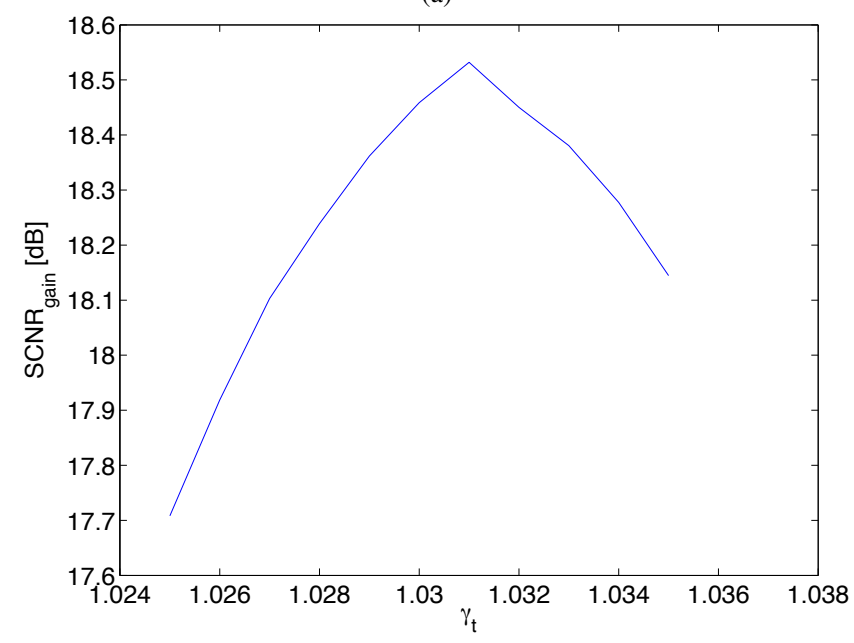

(b)

Fig. 11: In this figure, the SCNR of the target in the SAR image after focusing the target for different normalized relative speed is shown. In Fig. 11a, the step size is 0.01 , and the measurements span from 0.96 to 1.04 . At 1.03 there is a clear peak. A smaller step size of 0.001 is used around 1.03 and shown in Fig. 11b. At 1.031 a clear peak can be distinguished. For these plots, the target bearing was chosen to 120 degrees.

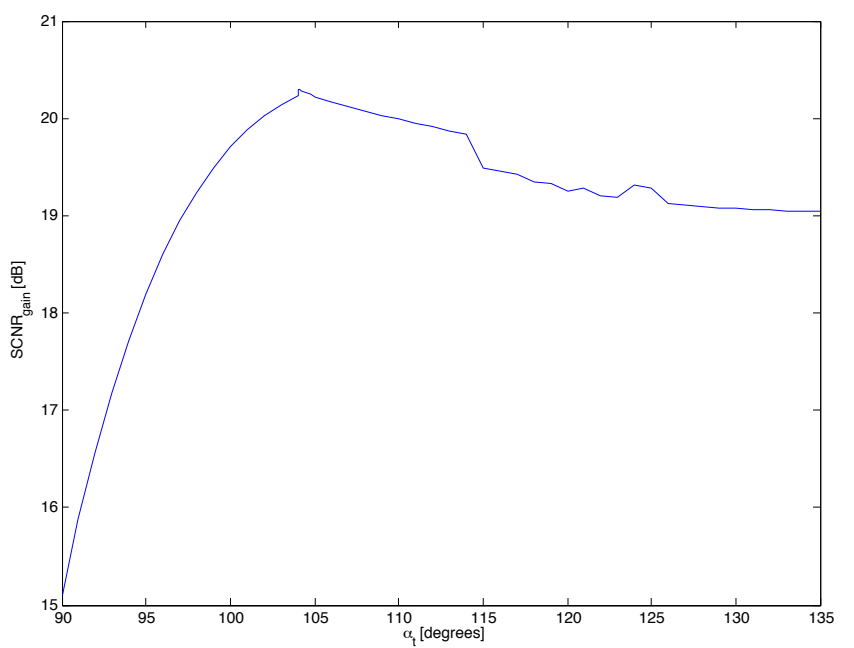

Fig. 12: In this figure, the SCNR of the target in the SAR image has been measured by searching in bearing, $\alpha_{t}$ from 90 to 135 degrees. The gain increases monotonically until 104 degrees, after which the gain is reduced gradually.

Refocusing in Synthetic Aperture Radar Images. IEEE Transactions on Aerospace and Electronic Systems, 48, 3 (2012), 2426-2436.

[25] Brennan, L.E., Reed, L.S. (1973) Theory of Adaptive Radar. IEEE Transactions on Aerospace and Electronic Systems, 9, 2 (1973), 237-252.

[26] Melvin, W.L. (2004) A STAP overview. IEEE Transactions on Aerospace and Electronic Systems, 19, 1 (2004), 19-735.

[27] Hellsten, H., and Ulander, L.M.H. (2000) Airborne array aperture UWB UHF radar - Motivation and system considerations IEEE Aerospace and Electronic Systems Magazine, 15, 5 (2000), 35-45.

[28] Ulander, L.M.H. and Hellsten, H. (1999) Low-frequency ultra-wideband array-antenna SAR for stationary and moving target imaging In Proceedings of Radar Sensor Technology IV, held in Orlando, FL, 5-9 April 1999, SPIE 3704 (1999), 149-158.

[29] Ulander, L.M.H. (1991) Accuracy of using point targets for SAR calibration. IEEE Transactions on Aerospace and Electronic Systems, 28, 1 (1991), 139-148.
[19] Cerutti-Maori, D., Sikaneta I. (2010) Optimum GMTI processing for space-based SAR-GMTI systems: Simulation results. Proceedings of EUSAR, Aachen, Germany, 7-10 June (2010).

[20] Ulander, L. M., Blom, M., Flood, B., Follo, P., Frölind, P.-O., Gustavsson, A., Jonsson, T., Larsson, B., Murdin, D., Pettersson, M., Rääf, U., Stenström, G. (2003) The VHF/UHF-band LORA SAR and GMTI System. In Proceedings of SPIE Algorithms for Synthetic Aperture Radar Imagery X, Orlando, FL, 21-23 April, (2003).

[21] Yegulalp, A.F. (1999) Fast backprojection algorithm for synthetic aperture radar. In IEEE Radar conference, Waltham, MA, 20-22 April, (1999).

[22] Ulander, L.M.H., Hellsten, H., Stenström, G . (2003) Synthetic-aperture radar processing using fast factorized back-projection. In IEEE Transactions of Aerospace and Electronic Systems, 39, 3 (2003), 760-776.

[23] Frolind, P.-O., Ulander, L.M.H. (2006) Evaluation of angular interpolation kernels in fast back-projection SAR processing. In IEE Radar, Sonar and Navigation, 153, 3 (2006), 243-249.

[24] Sjögren, T.K., Vu, V.T., Pettersson, M.I., Gustavsson, A, Ulander, L.M.H. (2012) Moving Target Relative Speed Estimation and 


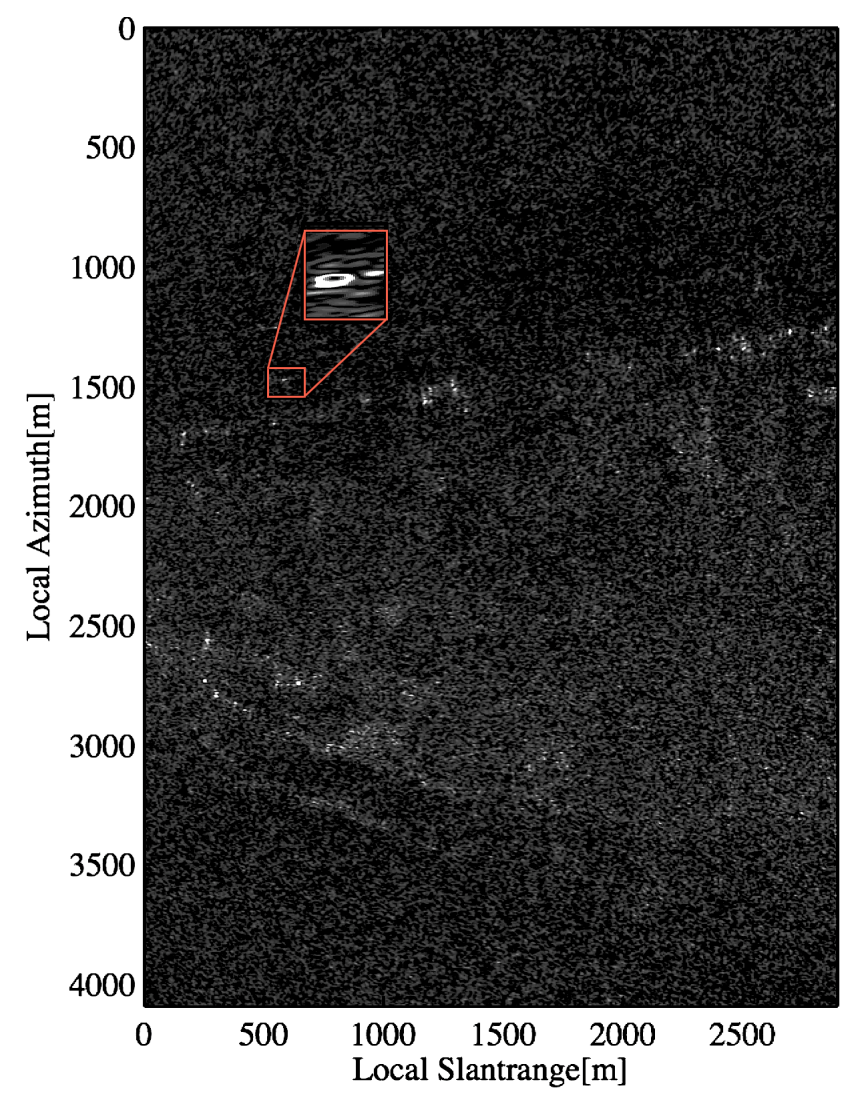

Fig. 13: In this figure, the SAR image after focusing the target for relative speed is shown. The target signature is highlighted with a red square and a zoom-in can be viewed. It must be noted that the truck is now the strongest of all reflections in the SAR image. Even the very strong specular reflections found in the original image are successfully suppressed to the noise level and can no longer be detected in the image. 